\title{
Estudios taxonómicos en el género Festuca L. (Poaceae) de Argentina y Chile
}

\section{Taxonomic studies in the genus Festuca L. (Poaceae) from Argentina y Chile}

\author{
Juan Camilo Ospina González, Sandra S. Aliscioni \& Silvia S. Denham* \\ Instituto de Botánica Darwinion (ANCEFN-CONICET), Labardén 200, Casilla de Correo 22, B1642HYD San Isidro, Buenos \\ Aires, Argentina. \\ *sdenham@darwin.edu.ar
}

\begin{abstract}
RESUMEN
En esta contribución se analiza la variación morfológica en el complejo de especies relacionadas a Festuca chrysophylla por medio de análisis estadísticos multivariados de caracteres morfológicos y anatómicos. Se describe la morfología de $F$. chrysophylla, se establecen sus sinónimos y se actualiza su distribución geográfica. Se establece un nuevo sinónimo: $F$. orthophylla bajo $F$. chrysophylla. Asimismo, sobre la base del estudio de material de herbario se registra por primera vez la presencia de Festuca werdermannii para Argentina, hasta ahora sólo conocida para Chile. Se describe detalladamente la anatomía foliar para ambas especies sobre la base del estudio de numerosos ejemplares. Se seleccionan lectotipos para $F$. chrysophylla y para $F$. deserticola var. juncea.
\end{abstract}

Palabras clave: Flora Cono Sur, análisis multivariados, anatomía, morfología.

\begin{abstract}
In this contribution we analyze the morphological and anatomical variation in the complex of species related to Festuca chrysophylla using multivariate analysis. We describe the morphology of $F$. chrysophylla, synonyms are established and the geographical distribution is updated. We establish one new synonymy: F. orthophylla under F. chrysophylla. Furthermore, based on herbarium material, we provide the first record for $F$. werdermannii for Argentina. We describe in detail the anatomy of both species based on the study of numerous specimens. We select lectotypes for $F$. chrysophylla and $F$. deserticola var. juncea.
\end{abstract}

KEYwords: Southern Cone Flora, multivariate analysis, anatomy, morphology.

\section{INTRODUCCIÓN}

El género Festuca L. reúne 450-500 especies en todo el mundo, de las cuales 150-200 han sido citadas para América del Sur y 56 para el Cono Sur de América; crecen en regiones templadas y templado-frías de ambos hemisferios (Soreng et al. 2003, Stančík \& Peterson 2007, Zuloaga et al. 2008, Catalán \& Müller 2012).

El género Festuca ha sido estudiado para América del Sur (St.-Yves 1927, Hitchcock 1927, Macbride 1936, Stančík \& Peterson 2007) para floras regionales de Argentina (Stuckert 1906, Parodi 1935, 1953, Türpe 1969, 1998 Nicora 1978, Nicora \& Rúgolo 1987, Catalán \& Müller 2012), Chile (Matthei 1982, Marticorena \& Quezada 1985, Finot et al. 2009), Bolivia (Renvoize 1998), Perú (Infantes 1952, Tovar 1972), Ecuador (Alexeev 1986, Laegaard 1999); Colombia
(Alexeev 1984, Rangel Churio et al. 1997, Rangel Churio 2000, Stančík 2003a, 2003b); y Venezuela (Alexeev 1986, Briceño \& Morillo 1994, Stančík 2001).

Debido al amplio rango de variación morfológica que presentan los taxones de Festuca, la delimitación de las especies es dificultosa, por lo tanto, los caracteres anatómicos de las láminas han sido tradicionalmente usados para colaborar en la delimitación de taxones. Sin embargo, también son caracteres insuficientes o ambiguos en muchos casos. La falta de caracteres morfológicos y anatómicos estables ha resultado en que diferentes autores usen distintos criterios para reconocer a las especies, o que, en un mismo tratamiento, los límites específicos frecuentemente se superpongan. Esto dificulta establecer caracteres diagnósticos para las especies del género, incluso para las especies de un área delimitada. 
La aplicación de técnicas numéricas multivariadas a complejos de especies o a taxones morfológicamente afines contribuye a reconocer grupos discretos de especímenes con mayor objetividad y a detectar los caracteres que permiten separarlos. Estas técnicas, sumadas al estudio detallado de los materiales tipo y descripciones originales, han sido ampliamente usadas para resolver grupos de especies conflictivas (Henderson 2005, Denham \& Aliscioni 2010, Escobar et al. 2011).

Matthei (1982) cita para Chile 28 especies de Festuca y Catalán \& Müller (2012) 46 para Argentina. Si bien estos trabajos constituyen importantes revisiones, persisten dificultades en reconocer los taxones que crecen en estos países. En el marco de la revisión del género Festuca para la flora de Chile, se detectó la necesidad de establecer límites precisos en el complejo "Festuca chrysophylla" que incluye a: Festuca chrysophylla Phil., F. eriostoma Hack. y F. orthophylla Pilg.; y ampliar la distribución de $F$. werdermanii St.-Yves, hasta ahora endémica de Chile.

El objetivo de esta contribución es delimitar las especies del complejo "Festuca chrysophylla" aplicando análisis estadísticos multivariados, sobre la base del estudio de material de herbario. Se circunscriben las especies, se proponen nuevos sinónimos y se actualizan los datos de distribución geográfica de acuerdo a los límites establecidos para los taxones aquí estudiados. Se registra una cita nueva para la flora de Argentina.

COMPLeJo FestuCA CHRYSOPHYLla

Este complejo incluye tres taxones considerados válidos (Festuca orthophylla, F. chrysophylla y F. eriostoma) y nombres considerados actualmente sinónimos, según Soreng et al. (2003) y Zuloaga et al. (2008): F. orthophylla var. boliviana Pilg., F. orthophylla var. glabrescens Pilg., F. orthophylla var. meyenii St.-Yves $(=F$. orthophylla) y $F$. juncea Phil., F. paupera Phil. y Festuca saltana St.-Yves (= F. Chrysophylla).

El complejo "Festuca chrysophylla" constituye un grupo de taxones morfológicamente definido por láminas foliares de transcorte conduplicado, superficie adaxial densamente piloso-pubescente, con pelos que sobresalen por el margen foliar formando una línea blanquecina, la superficie abaxial es pilosa en la base y glabra hacia el ápice. Las láminas son de hasta $20 \mathrm{~cm}$ de largo, juncoides y sumamente rígidas, erectas a divergentes. Presentan panículas de (8)10-20 cm largo, con ramas adpresas al raquis, las espiguillas presentan lemas pilosas a densamente pubescentes en el margen y el ápice mucronulado.

Las especies de este complejo se distribuyen en Perú, Bolivia, hasta el centro de Chile y Argentina. Habitan en suelos salinos, orillas de arroyos, vegas y laderas rocosas de la Puna y regiones andinas, entre 3.225 y $4.860 \mathrm{~m}$ de altitud. Constituyen importantes pajonales serranos (Stuckert 1906) y son importantes componentes de la asociación "Festuca orthophylla", "Festuca chrysophylla" y "Poa gymnantha" de la Puna argentina, donde forman extensas matas xerófilas bajas y punzantes, notables por su color dorado-cobrizo (Cabrera 1957).

Türpe (1969) establece que en la Argentina habitan tres taxones de este complejo: F. orthophylla, F. eriostoma y $F$. chrysophylla y menciona la afinidad que presentan estas especies. Sin embargo, separa a $F$. eriostoma de F. orthophylla por su porte menor, su lígula netamente biauriculada, láminas provistas de base callosa y el raquis y ramas de la inflorescencia glabros. Además, usa la pilosidad del ovario y la anatomía foliar para separar a $F$. chrysophylla de las demás (con haces semitrabados en F. chrysophylla y con al menos tres haces trabados en las restantes).

Tovar (1972), en la revisión de las especies peruanas del género Festuca, reconoce dos variedades de F. orthophylla: la variedad tipo y la var. glabrescens. Los caracteres que usa este autor para diferenciarlas son: la pubescencia de lemas y glumas, el largo de la panícula, la pilosidad de las ramas de la panícula y de las vainas. Este autor no cita a F. eriostoma ni F. chrysophylla para Perú.

Matthei (1982) trata las especies de Festuca para Chile y cita a $F$. orthophylla y $F$. chrysophylla. Este autor las diferencia por la pilosidad de la lema ( $F$. orthophylla presenta lemas pilosas y $F$. chrysophylla, lemas glabras). Sin embargo, menciona que en ejemplares de $F$. chrysophylla la pilosidad de la lema puede ser variable y asemejarse a F. orthophylla. Según el mismo autor, ambas especies coinciden en sus caracteres anatómicos: presentan un contorno foliar redondeado o elíptico, haces trabados (1 ó 2 haces trabados abaxialmente) y epidermis abaxial con células cortas solitarias y presencia de células suberosas.

Renvoize (1998), para la flora de Bolivia, cita a $F$. eriostoma, F. chrysophylla y $F$. orthophylla. Considera que F. eriostoma podría sinonimizarse con $F$. orthophylla, ya que sólo se diferencia por su menor altura y láminas foliares patentes o deflexas. En la clave utiliza el color de las láminas para diferenciar a $F$. chrysophylla y $F$. orthophylla (anaranjadas vs. glaucas o verdosas, respectivamente).

Catalán \& Müller (2012) reconocen para la flora argentina a $F$. orthophylla y a $F$. chrysophylla. Incluye a F. eriostoma como sinónimo de F. orthophylla. Separan a las dos especies válidas por el hábito (F. orthophylla como plantas cespitosas con cortos rizomas vs. F. chrysophylla plantas cespitosas, sin rizomas), por la forma y anatomía de las láminas (hojas junciformes, de 1-1,2 mm de diámetro, con trabéculas completas de esclerénquima en nervios primarios y secundarios en F. orthophylla vs. hojas setáceas, de 0,6-0,7 $\mathrm{mm}$ de diámetro, con trabécula completa de esclerénquima únicamente en el nervio medio en F. chrysophylla) y por la pilosidad del ápice del ovario (glabro vs. esparcidamente piloso en F. chrysophylla).

Los caracteres usados por diferentes autores para la delimitación de las especies del complejo "Festuca 
chrysophylla" difieren entre sí y, en algunos casos, son ambiguos. Además, se encontraron ejemplares determinados bajo distintos nombres por diferentes autores. Por las mismas razones, los datos sobre la distribución geográfica de los taxones son también imprecisos. Por lo tanto, es necesario delimitar claramente las especies, esclarecer la taxonomía y documentar con mayor precisión la distribución geográfica de las especies del complejo.

\section{MATERIALES Y MÉTODOS}

Morfología

Se consultaron colecciones de los herbarios BAA, BAB, CONC, LIL, LP, LPB, SI, SGO (Thiers 2012). Se estudiaron ejemplares tipo depositados en CONC, SGO y SI e imágenes digitales disponibles en línea. Se revisaron 180 ejemplares; para el análisis estadístico fueron seleccionados 100 especímenes completos y determinados como $F$. orthophylla (55 ejemplares), F. chrysophylla (25) y F. eriostoma (20). De los anteriores, 39 ejemplares se usaron para tomar datos anatómicos (F. orthophylla 20 ejemplares, F. chrysophylla 11, F. eriostoma 8). Se registró cada ejemplar de acuerdo a la última determinación hallada en la cartulina y a los ejemplares sin determinar se les asignó un nombre de acuerdo a Türpe (1969) por incluir una clave para las tres especies válidas en Zuloaga et al. (2008).

Cabe resaltar que numerosos ejemplares pertenecientes al complejo "Festuca chrysophylla" han sido determinados bajo el nombre Festuca lassiorrhachis Pilg. Sin embargo, el ejemplar tipo de este último taxón y su descripción original (Pilger 1906) difieren del patrón morfológico que presentan las especies del complejo aquí estudiado, ya que sus láminas son marcadamente amplias, glabras en la superficie adaxial, frágiles y las lemas aristadas. Aquí seguimos a Catalán \& Müller (2012) que consideran a esta especie bajo la sinonimia de F. dolichophylla J. Presl \& C. Presl.

Los caracteres morfológicos de vaina y lámina se tomaron de la tercera o penúltima hoja de macollos infértiles. Para los restantes caracteres se usó la estructura que reflejara la mayor expresión del atributo en cada ejemplar; así, la caña florífera más larga, la espiguilla con mayor número de antecios o la lígula de mayor longitud.

\section{AnATomíA}

Para la observación de rasgos anatómicos se utilizó el tercio medio de una lámina desarrollada de una innovación estéril. El material se hidrató con amoniaco $\left(\mathrm{NH}_{3}\right)$ y agua destilada por $48 \mathrm{~h}$ a temperatura ambiente y se fijo en GALA (glicerina, alcohol y ácido acético) por 24 horas (Ruzin 1999). Se realizaron cortes transversales a mano alzada, los cuales se decoloraron con hipoclorito de sodio diluido al $50 \%$, se lavaron con agua destilada y se tiñeron con safranina. Para los preparados de epidermis adaxial y abaxial en vista tipográfica, se siguió la técnica propuesta por Metcalfe (1960). Los preparados fueron montados en gelatina-glicerina. Para la descripción de caracteres anatómicos se siguió la terminología propuesta por Ellis (1976, 1979). Los ejemplares de los cuales se obtuvieron cortes anatómicos y preparados epidérmicos se señalan con asterisco $(*)$ bajo Especímenes examinados.

\section{ANÁLISIS ESTADÍSTICO}

El análisis de datos se llevó a cabo mediante la aplicación de métodos multivariados: análisis de conglomerados (AC) y análisis de coordenadas principales (ACop); ya que ambos permiten el uso de variables cualitativas y cuantitativas (Balzarini et al. 2008). Se usó el software estadístico Infostat ver. 2011 (Di Rienzo et al. 2011).

Se definieron 23 caracteres como variables (10 morfológicos y 13 anatómicos, Apéndice I). Se construyó una matriz sobre la base de 100 ejemplares y 10 caracteres morfológicos y otra matriz utilizando 39 ejemplares para 23 caracteres anatómicos y morfológicos. Las variables que no presentaron variación fueron eliminadas previamente del análisis. Se consideraron los caracteres diagnóstico para la identificación de las unidades taxonómicas citados en trabajos para Argentina y Chile, y en lo posible, los ejemplares citados en estos mismos, con el fin de representar el criterio taxonómico de los autores en cada trabajo.

Para la matriz basada en datos morfológicos se realizó el análisis de conglomerados con el algoritmo UPGMA (unweighted pair-groupmethod using an arithmetic average), utilizando el coeficiente de distancia Gower (Gower 1971). Se calculó el coeficiente de correlación cofenético (r). Posteriormente, se aplicó el ACoP utilizando el coeficiente de correlación de Pearson. Los datos fueron estandarizados sustrayendo de cada observación la media y dividiendo el resultado por la desviación estándar. Para la matriz basada en datos anatómicos y morfológicos se aplicó el ACoP siguiendo los pasos anteriormente descriptos para datos morfológicos.

\section{SinONIMIAS}

Se estudiaron los ejemplares tipo de todos los sinónimos citados por Soreng et al. (2003) y en Zuloaga et al. (2008) a través de la observación directa del ejemplar o de fotos digitales de alta calidad. Se recopilaron todas las descripciones originales. Cabe resaltar que se ubicó un isotipo que no se había citado, corresponde a F. orthophylla var. boliviana Pilg. depositado en el Herbario Nacional de Colombia-COL. Se compararon cada uno de los materiales tipo y/o sus descripciones originales con los datos de variación morfológica establecidos para $F$. chrysophylla con el fin de corroborar las sinonimias. 


\section{RESULTADOS}

COMPLEJO FestuCA CHRYSOPHYLLA

Los análisis estadísticos mostraron que los atributos morfológicos y anatómicos analizados reúnen ejemplares de F. chrysophylla, F. eriostoma y F. orthophylla sin discernimiento de grupos que se correspondan con alguna de las entidades taxonómicas (Fig. 1). Esto sugiere similitud entre los atributos de estos taxones, lo cual fue señalado en otros trabajos (Türpe 1969, Renvoize 1998, Catalán \& Müller 2012).

El análisis de conglomerados muestra que los ejemplares de las tres especies se reúnen en grupos heterogéneos y no muestran un patrón que coincida con las especies estudiadas. El fenograma (Fig. 1C) (índice de correlación cofenético $=0,77$ que indica una buena representación de la matriz de distancia) muestra dos grandes conglomerados separados a distancia 0.53 que reflejan la semejanza entre las tres unidades taxonómicas. Estos grupos se caracterizan por reunir ejemplares de los tres taxones, inclusive, en los subgrupos y divisiones más finas del fenograma.

El análisis de coordenadas principales con datos morfológicos (100 especímenes, Fig. 1B) muestra que los ejemplares se distribuyen aleatoriamente en el espacio bidimensional. Los grupos apenas definidos que podrían observarse están constituidos por especímenes de los tres taxones. El análisis con datos anatómicos y morfológicos (39 especímenes, Fig. 1C) muestra el mismo resultado, indicando que las características anatómicas tampoco permiten separar a las entidades estudiadas.

El alto de la planta fue empleado por Türpe (1969) y por Renvoize (1998) para separar F. eriostoma (hasta 20 ó $30 \mathrm{~cm}$ de alto) y separarla de $F$. orthophylla (mayor de 20 ó $30 \mathrm{~cm}$ de alto). Sin embargo, se observó que este carácter es variable en ejemplares de un mismo taxón y forma un continuo en el total de ejemplares analizados.

Türpe (1969) y Nicora (1978) mencionan que la presencia de pelos en el ápice del ovario es un carácter difícil de observar con precisión, ya que son muy escasos y se desprenden con facilidad. En este trabajo se corrobora esta característica de los pelos fácilmente caducos $\mathrm{y}$, en muchos casos, la falta de observación de pelos en el ápice del ovario podría deberse a que los mismos se desprendieron, y se pone en duda la constancia de este carácter dentro de una especie.

El carácter de rizoma corto o ausente usado por Catalán \& Müller (2012) es, al menos, difícil de establecer en todos los ejemplares, aunque se cuente con especímenes completos. Todos los ejemplares observados son perennes, cespitosos, con un rizoma muy corto o apenas desarrollado que permite matas más flojas, pero no observamos un punto que permita delimitar dos estados para este carácter.
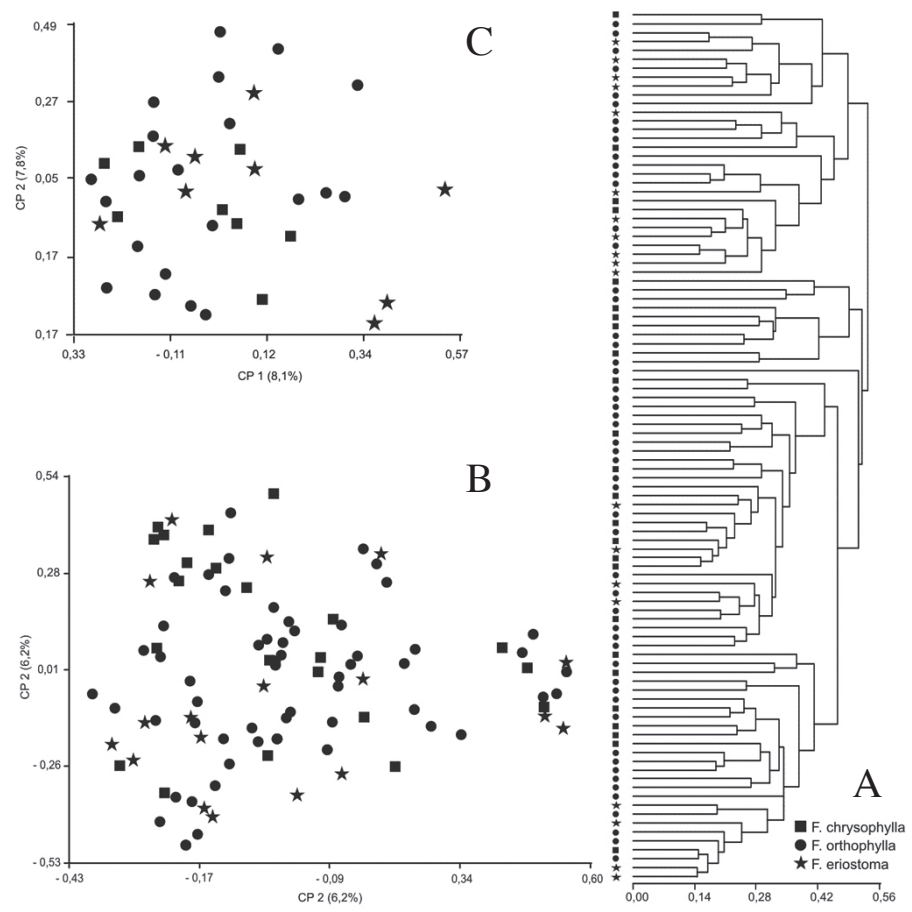

Figura 1. Análisis estadísticos multivariados. A. ACoP, proyección de 39 ejemplares basado en datos morfológicos y anatómicos; B. ACoP, proyección de 100 ejemplares basado en datos morfológicos. C. AC, fenograma basado en 100 ejemplares y datos morfológicos.

Figure 1. Multivariate analyses. A. PcoA, plot based on 39 specimens from the morphological and anatomical data; B. PcoA, plot based on 100 specimens from the morphological data; C. CA, pfhenogram based on 100 specimens from the morphological data. 
En cuanto a la anatomía, se observó que todos los ejemplares analizados del complejo "Festuca chrysophylla" presentan transcorte plegado, conduplicado, con un anillo esclerenquimático continuo subepidérmico en la cara abaxial, también observados por otros autores (Türpe 1969, Matthei 1982, Catalán \& Müller 2012). En relación a los haces vasculares y la presencia de trabas esclerenquimáticas adaxiales y abaxiales, se observó que existe variación y no se correlacionan a lo establecido por Türpe (1969), Matthei (1982) y Catalán \& Müller (2012) para diferenciar los taxones de este complejo.

Las láminas constan de 3 a 5 haces vasculares primarios que alternan con haces menores, el haz primario central presenta generalmente trabas esclerenquimáticas adaxial y abaxial, excepto algunos ejemplares como Peterson 12808 y Ayala 4 que sólo presentan traba abaxial.

Los haces primarios contiguos al central pueden variar en cuanto al desarrollo de las trabas esclerenquimáticas: pueden presentarse igual al haz primario central, es decir con trabas adaxial y abaxial, al menos uno de ellos, como por ejemplo Percy 7627 (F. orthophylla), Cabrera 30528 ( $F$. eriostoma) y Zuloaga 9180 (F. chrysophylla); o solamente con traba esclerenquimática abaxial como en Cabrera 32486 (F. orthophylla); o pueden ser haces libres o sólo con trabas adaxiales como por ejemplo Beck 23694 (F. orthophylla). Corroboramos así que, al analizar un número considerable de especímenes, los caracteres anatómicos usados por otros autores como diagnósticos no se mantienen estables dentro de las especies consideradas en el complejo.

Nueva cita para la Flora de Argentina

Festuca werdermannii es una especie descripta por SaintYves (1927) para Chile. Luego de su descripción, sólo ha sido citada para la Flora de Chile (Alexeev 1984; Matthei 1982, Marticorena y Quezada 1985). Para la Argentina no se había registrado su presencia. En el marco de la revisión de Festuca para la Flora de Chile, se encontraron ejemplares recolectados en Argentina cuyas características se corresponden con las de $F$. werdermannii y ésta constituye la primera cita para ese país.

Los especímenes argentinos se hallaron erróneamente determinados bajo los nombres Festuca argentinensis (St.Yves) Türpe; F. hypsophila Phil.; F. acanthophylla E. Desv.; F. villipalea (St.-Yves) E.B. Alexeev ( $=$ F. dissitiflora Steud. ex Griseb.); F. weberbaueri Pilg.; y F. nardifolia Griseb.; sin embargo, estas especies pueden diferenciarse claramente de F. werdermannii por los caracteres de la Tabla I.

\section{TRATAMIENTO TAXONÓMICO}

Festuca chrysophylla Phil., Verz. Antofagasta Pfl. 88. 1891. Festuca deserticola Phil. var. chrysophylla (Phil.) St.-Yves, Candollea 3: 212. 1927. TIPO: Chile. Incahuasi, 22 feb. 1885, F. Philippi s.n. [lectotipo aquí designado SGO-68814!, duplicados LP-290542!, SGO-62721!, SGO-37422!, US556557, foto!, W-1916-0039766, foto!]. Fig. 2.

Festuca orthophylla Pilg., Bot. Jahrb. Syst. 25(5): 717. 1898, syn. nov. TIPO: Perú. Viconcaya, "in vicinitae oppidi Arequipa", 1 feb. 1877, A. Stübel 87 [holotipo B-4726b, foto!; isotipos HP no visto, US-81579, foto!].

Festuca eriostoma Hack., Oesterr. Bot. Z. 53: 32. 1903. Festuca orthophylla Pilg. var. eriostoma (Hack.) St.Yves, Candollea 3: 195. 1927. TIPO: Argentina. La Rioja, Sierra de Famatina, entre la Mina Yareta y la altura de Espíritu Santo, 26 ene. 1879, G. H. E.Hieronymus \& G. Niederlein 781 (lectotipo W no visto, foto!, designado por Alexeev, Bot. Zhürn. 69: 348. 1984; duplicados B-4704, foto!, JE-00004960, foto!, K no visto, S-R-2262, foto!, CORD00001639, foto!, CORD00001640, foto!, US2875407, foto!).

Festuca orthophylla Pilg. var. glabrescens Pilg., Bot. Jahrb. Syst. 37: 507. 1906. Festuca orthophylla Pilg. subvar. glabrescens (Pilg.) St.-Yves, Candollea 3: 197. 1927. TIPO: Perú. "Ad vicum Pucará in via Ferrea inter oppida Puno et Cuzco, in pratis apertis frequentissima", $3700 \mathrm{~m}$, feb. 1902, A. Weberbauer 408 (lectotipo B-10-0250137,

TABLa I. Atributos que permiten separar a Festuca werdermannii St.-Yves de otros taxones.

TABLE I. Attributes that allow separate Festuca werdermannii St.-Yves. from other taxa.

\begin{tabular}{|c|c|c|c|c|}
\hline \multirow{2}{*}{ TAXONES } & \multicolumn{4}{|c|}{ CARACTERES } \\
\hline & LARGO LÁMINA $(\mathrm{cm})$ & LARGO LÍGULA (mm) & ÁPICE OVARIO & PÁlea, CARINAS \\
\hline F. werdermannii & $5-10$ & $3-5$ & híspido & glabras \\
\hline F. argentinensis & $12-15$ & 0,5 & glabro & villosas \\
\hline F. hypsophila & $14-20$ & $0,3-0,5$ & glabro & villosa \\
\hline F. acanthophylla & $12-25$ & $0,5-1$ & pelos ralos & ciliadas \\
\hline F. dissitiflora & $11-18$ & ca. 0,5 & glabro & pilosas \\
\hline F. weberbaueri & $15-28$ & ca. 0,5 & glabro & ciliadas \\
\hline F. nardifolia & $2-5$ & 0,5 & glabro & ciliadas \\
\hline
\end{tabular}




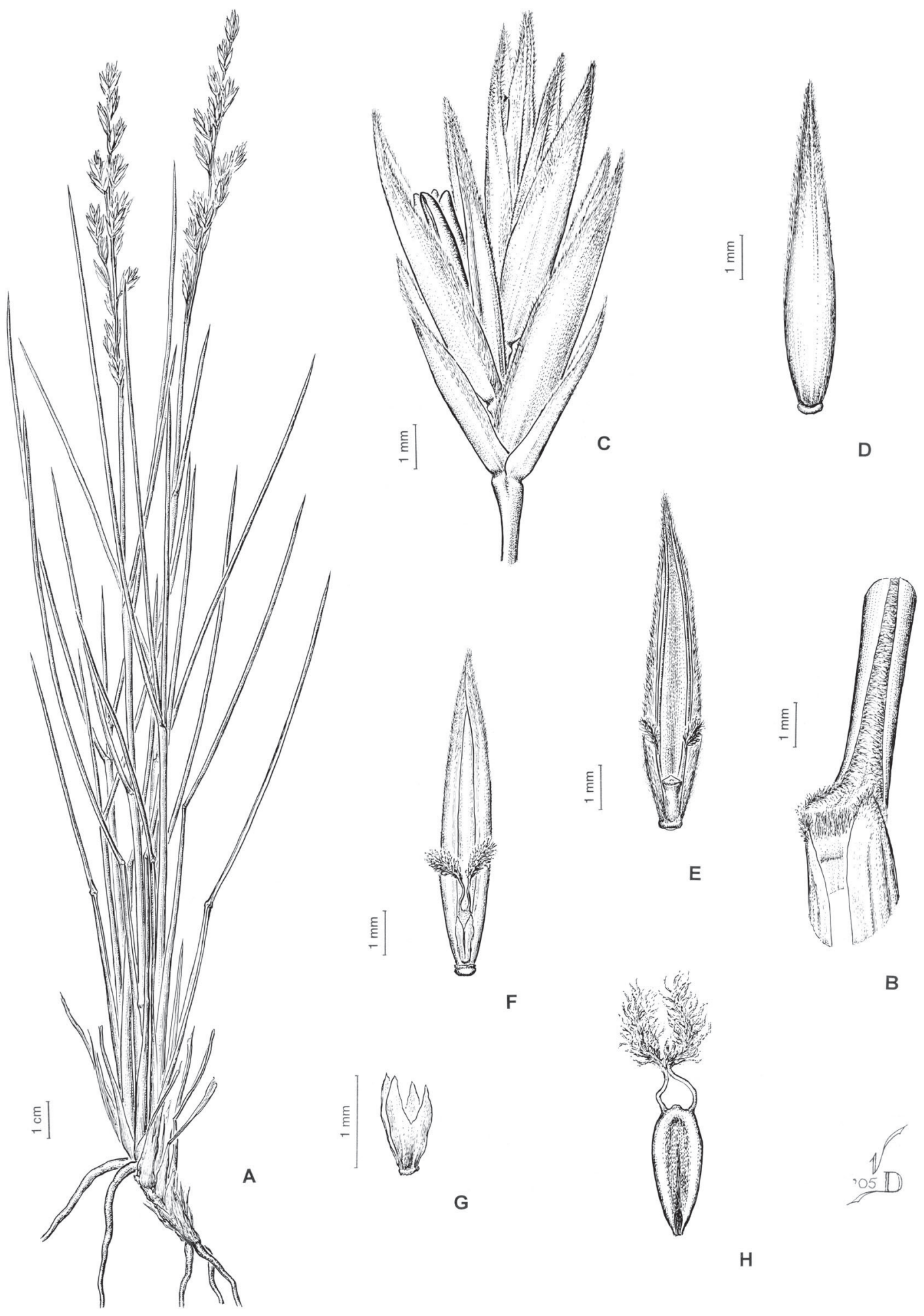

Figura 2. Festuca chrysophylla Phil., Cabrera et al. 30528 (SI). A. Hábito; B. Zona lígular y detalle de pelos sobresaliendo por el margen; C. Espiguilla; D. Antecio vista dorsal; E. Antecio vista ventral; F. Palea con lodículas y ovario; G. Lodículas; H. Ovario.

Figure 2. Festuca chrysophylla Phil., Cabrera et al. 30528 (SI). A. Habit; B. Ligular zone and hairs out of the margin; C. Spikelet; D. Anthecium dorsal view; E. Anthecium ventral view; F. Palea with lodicules and ovary; G. Lodícules; H. Ovary. 
foto!, designado por Alexeev, Bot. Zhurn. 69: 348. 1984; isolectotipo S-R-2290, foto!).

Festuca orthophylla Pilg. var. boliviana Pilg., Bot. Jahrb. Syst 37: 508. 1906. Festuca orthophylla Pilg. subvar. boliviana (Pilg.) St.-Yves, Candollea 3: 196. 1927. TIPO: Bolivia. In Puna Patanca, magnas altiplanitiei partes occupans, 3700 m, mar. 1904, K. Fiebrig 3192 (holotipo B-10-0250138, foto!; isotipos HP no visto, US-81578, foto!, COL!, foto K000433593!, foto K000433594!, foto S054913!, foto S-R-2289!).

Festuca orthophylla Pilg. var. meyenii St.-Yves, Candollea 3: 198. 1927. Festuca meyenii (St.-Yves) E.B. Alexeev, Bot. Žhurn. (Moscow \& Leningrad) 69: 348. 1984. TIPO: Perú. "Ad pedem M. igni. Arequipa", $13000 \mathrm{ft}$, abr. 1831, Meyen s.n. (lectotipo B no visto, designado por Alexeev, Bot Zhurn. 69: 348. 1984).

Festuca deserticola var. juncea St.-Yves, Candollea 3: 209. 1927. Festuca juncea Phil., Verz. Antofagasta Pfl. 88, 89. 1891, nom. illeg. hom., non Festuca juncea (L.) Moench. TIPO: Chile. "Aguas calientes et Socaire”, 3200 m, F. Philippi s.n. [lectotipo aquí designado SGO-63822!, isotipos SGO-37433!, SGO-62724!, W-1916-0039745, foto!, US-556551, foto! (fragm. ex SGO-PHI-294 \& foto)].

Hierba perenne, densamente cespitosa, raras veces desarrolla el rizoma. Cañas floríferas hasta de $70 \mathrm{~cm}$ de alto. Vainas abiertas, pilosas en la parte superior, coriáceas, pajizas a blanquecinas, cuello truncado. Pulvínulo emergente, glabro. Lígulas 0,3-1 mm de largo, truncadas, membranáceo-ciliado, pubescentes. Láminas de $8-20 \mathrm{~cm}$ de largo, juncoides, sumamente rígidas, erectas a divergentes, superficie abaxial glabrescente, superficie adaxial densamente pubescente (los pelos sobresalen por su margen, formando una línea blanquecina). Panículas (8) 10-20 cm de largo, lanceoladas; ramas adpresas al raquis, esparcidamente pilosas a glabrescentes. Espiguillas 8-10 mm de largo, 4-6 floras, raquilla pilosa. Glumas que no superan el medio de los antecios contiguos, escariosas, ciliadas en margen y ápice; la inferior 4,4-5,2 $\mathrm{mm}$ de largo, linear, 1 nervia; la superior 6-7 mm de largo, lanceolada, 3 nervia. Lema 5-6,8 $\mathrm{mm}$ de largo, densamente pilosa en el margen, ápice mucronulado. Pálea $6,2 \mathrm{~mm}$ de largo, herbácea, pajiza a blanquecina, carenas pilosas. Anteras 3-5,5 mm de largo. Lodículas 0,8-1 $\mathrm{mm}$ de largo, margen fimbriado, glabras. Ovario glabro en el ápice. Cariopse ovoide. Número cromosómico: $2 \mathrm{n}=42$ (Dubcovsky \& Martínez 1991, bajo F. chrysophylla).

Nombres vulgares: Iru, Iro, Ichu, Paja de puna.

Caracteres histofoliares en corte transversal (Fig. 3 A y B). Láminas permanentemente plegadas, conduplicadas, de transcorte circular a elíptico, semiláminas simétricas, en algunos casos asimétricas (Ayala 4), de 0,5-1 mm de diámetro; superficie adaxial con costillas marcadas, redondeadas a agudas, asociadas a haces primarios, o en algunos casos también a secundarios, surcos notorios entre haces vasculares o sobre haces menores; superficie abaxial lisa; haces vasculares distribuidos aproximadamente equidistantes de ambas epidermis, 3-5(-7) haces primarios alternando con haces menores, todos los haces vasculares rodeados por una vaina esclerenquimática continua de células engrosadas en paredes radiales y tangenciales internas, en algunos casos con una segunda vaina irregular de células parenquimáticas pequeñas de paredes delgadas que se diferencian por su tamaño del resto del mesófilo; esclerénquima abaxial subepidérmico desarrollado, formando un anillo continuo constituido por 2-12 capas de células que se extiende hacia los márgenes, $\mathrm{y}$ forma trabas asociadas a todos o algunos haces primarios, esclerénquima adaxial escaso formando delgados casquetes o formando trabas delgadas asociadas a algunos haces vasculares; epidermis abaxial en corte transversal de células poco a muy engrosadas (Percy 7627; Peterson $15568,15508)$, de igual o mayor diámetro que la epidermis adaxial, sin asperezas, o cuando presentes abundantes (Cabrera 31849, 30528; Zuloaga 11179), epidermis adaxial con células de pared tangencial externa plana a algo convexa engrosada, con asperezas y estomas ubicados en las zonas de los surcos, macropelos unicelulares abundantes en zonas costales e intercostales, células buliformes escasamente diferenciadas en la profundidad de los surcos.

CARACteres DE la ePidermis Foliar EN Vista topográfica. Epidermis abaxial con zonas intercostales y costales no diferenciadas, células largas y cortas de contorno rectangular, de longitud variable, con paredes anticlinales longitudinales paralelas, muy engrosadas, con ondulaciones muy profundas, paredes anticlinales transversales paralelas, engrosadas y rectas; aparatos estomáticos ausentes; asperezas (ganchos y/o aguijones) ausentes o presentes, cuando presentes de moderados a abundantes, de base oval; micropelos ausentes; macropelos ausentes; cuerpos silíceos costales e intercostales solitarios alternando con células largas, verticalmente elongados, rectangulares o más estrechos en la zona media. Epidermis adaxial con zonas costales e intercostales bien diferenciadas, células largas de disposición y tamaño semejante a la epidermis abaxial, en algunos casos con paredes menos sinuosas y ligeramente más delgadas, aparatos estomáticos siempre presentes en zonas intercostales, con células subsidiarias de paredes redondeadas, en forma de domo bajo; asperezas presentes, en particular con mayor densidad en las zonas costales, semejantes a las de la epidermis abaxial; cuerpos silíceos costales e intercostales semejantes a los de la epidermis abaxial, macropelos siempre presentes, unicelulares, de base simple, rectos a ligeramente curvados, de 0.5-1 mm de largo. 

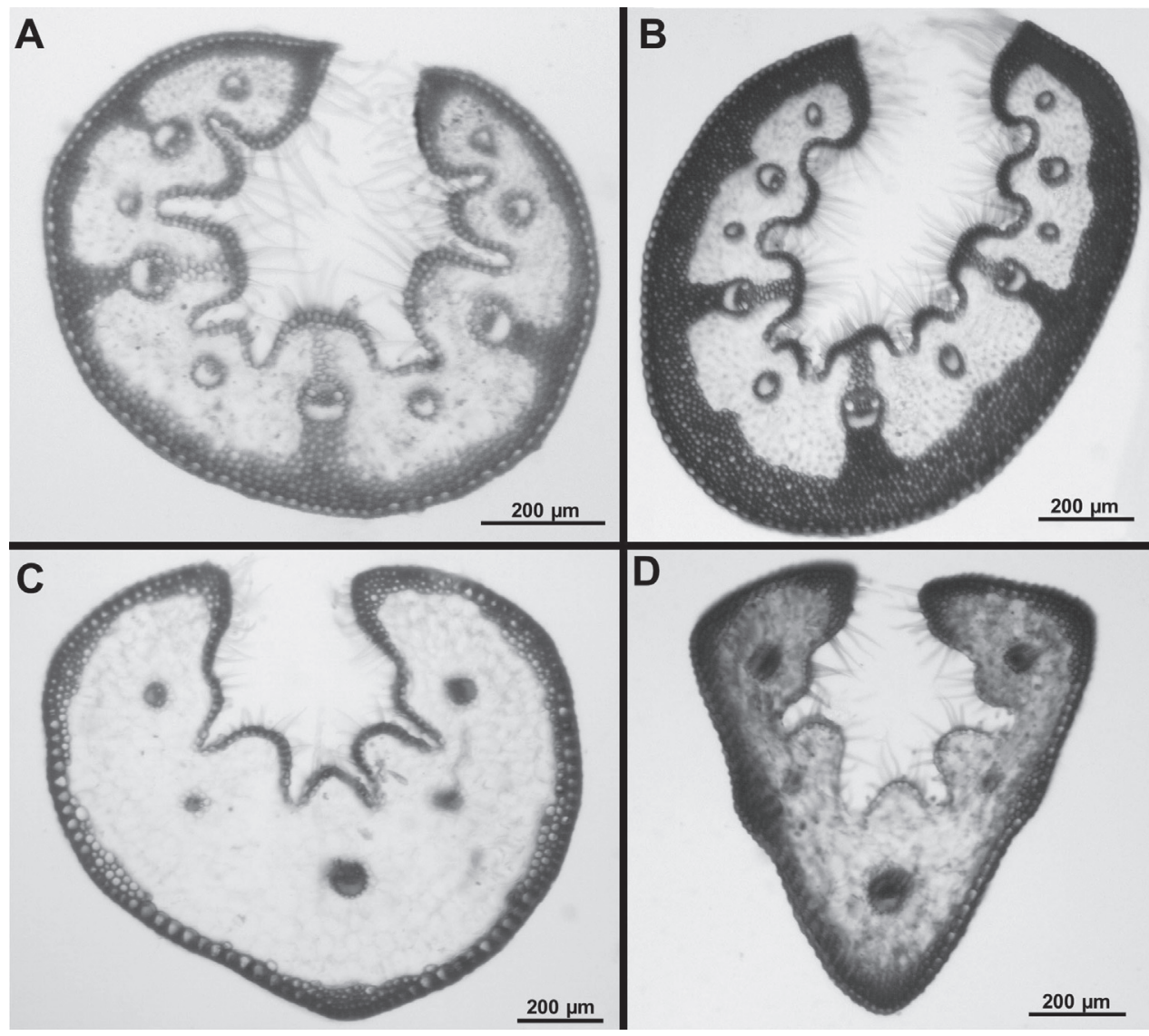

Figura 3. Cortes transversales de lámina foliar. A. Festuca chrysophylla Phill., Cabrera et al. 30528 (SI); B. Festuca chrysophylla Phil., Gómez-Kopta 92 (SI); C. Festuca werdermannii St.-Yves, Teillier 4526 (SI); D. Festuca werdermannii St.-Yves, Cabrera 3521 (SI).

Figure 3. Transverse sections of leaf blade. A. Festuca chrysophylla Phill., Cabrera et al. 30528 (SI); B. Festuca chrysophylla Phil., Gómez-Kopta 92 (SI); C. Festuca werdermannii St.-Yves, Teillier 4526 (SI); D. Festuca werdermannii St.-Yves, Cabrera 3521 (SI).

Distribución y hábitat (Fig. 4). Se distribuye en Perú, Bolivia, Argentina y Chile (entre $10^{\circ} 35^{\prime}$ y $30^{\circ} 48^{\prime}$ de latitud Sur) entre 3.100 y $4.900 \mathrm{~m}$ de altitud. Crece a lo largo de la Cordillera de los Andes y habita en ambientes xerofíticos, suelos salinos, orillas de arroyos, vegas y laderas rocosas de la Puna y regiones andinas. Constituyen importantes pajonales serranos (Stuckert 1906).

OBSERVACIONES: Esta especie se caracteriza por sus láminas juncoides y sumamente rígidas, erectas a divergentes, superficie adaxial densamente piloso-pubescente, con pelos que sobresalen por el margen foliar formando una línea blanquecina, la superficie abaxial es pilosa en la base y glabra hacia el ápice de transcorte conduplicado. Presentan panículas de 10-20 cm largo, con ramas adpresas al raquis, las espiguillas presentan lemas pilosas a densamente pubescentes en el margen y el ápice mucronulado.

En el protólogo de F. chrysophylla, R. A. Philippi menciona un ejemplar de Incahuasi de la colección de F. Philippi. Aquí designamos el ejemplar F. Philippi s.n.
(SGO-68814) ya que el mismo corresponde a esa localidad y fue coleccionado por Federico Philippi. El ejemplar erróneamente publicado como holotipo en Tropicos (www. mobot.org) y Catalán \& Müller (2012), R.A. Philippi s.n. (SGO-Phil-297), es de la localidad de Tarapacá y no se ha localizado en SGO.

Otros duplicados de R. A. Philippi s.n. han sido citados como isotipos de F. chrysophylla pero corresponden a ejemplares de otras especies (SGO-37446 corresponde a $F$. deserticola; SGO-63827 corresponde a F. tunicata Phil.). Otros isotipos publicados para esta especie no han sido localizados en SGO (SGO-63814, SGO-63830).

Existen varios duplicados de la colección de F. Philippi de la localidad tipo determinados por R. A. Philippi como F. juncea. Entre ellos, se seleccionó SGO-63822 por corresponder a las características del protólogo. El ejemplar citado como holotipo en Tropicos y Catalán y Müller (2012), SGO-Phil-294, no ha podido ser localizado en SGO.

Se excluye de la sinonimia de $F$. chrysophylla a $F$. paupera (SGO-63813! y SGO-37446!) y a $F$. saltana 
(holotipo G-00099483, foto!) ya que los ejemplares tipo examinados no corresponden a F. chrysophylla.

Especímenes examinados: ARGENTINA, Prov. Catamarca, Depto. Antofagasta de la Sierra, Ruta 53, falda de La Ciénaga. 19-II-1980. Cabrera et al. 31849* (SI); Depto. Belén, Laguna Blanca, 3200 m. 01-IV-1982. Gómez-Kopta 69 (SI); 01-VIII-1981. Gómez-Kopta 92* (SI); Reserva de la Biosfera Laguna Blanca, 4200 m. 16-III-1989. Reca et al. 143 (SI); 3800 m. 16-III-1989. Reca et al. 41 (SI); 3150 m. 21-III-1989. Reca et al. 161 (SI); 3800 m. 16-XI-1989. Reca et al. 40 (SI); 3200 m. 15-III-1989. Reca et al. 52 (SI); 3600 m. 21-III-1989. Reca et al. 169 (SI); 3750 m. 20-III-1989. Reca et al. 196 (SI); vega cerca de Gendarmería Laguna Blanca, 3200-3300 m. 17-III-1989. Reca et al. 1 (SI); Ruta Prov. 43, entre villa Vil y Antofagasta de la Sierra, a $39 \mathrm{~km}$ de la primera, Quebrada de Randolfo, 3212 m. 25-IV-2006. Biurrun et al. 8297* (SI); dejando la Ruta Prov. 43 entre Villa Vil y Antofagasta de la Sierra, a $20 \mathrm{~km}$ del cruce con ésta, Laguna Blanca, 3290 m. 26-IV-2006. Biurrun 8319 (SI); Laguna Blanca. 24-II-1981. Cabrera et al. 32486* (SI); camino a Antofagasta, Pasto Ventura. 26-II-1981. Cabrera et al. 32548 (SI); cerca de Gendarmería, 3200-3300 m. 14-III1984. Reca et al. 25 (SI); Cuesta de Randolfo, 3400 m. 23-
III-1981. Cabrera et al. 32462 (SI); Depto. Santa María, Cerro Aconquija. 4500 m. 15-IV-1917. P. Jörgensen 1721 (SI); Depto. Tinogasta, Po. San Francisco, La Coipa, Rincón. 3600 m. 07-II-1995. Ruthsatz 9253* (SI). Prov. Jujuy, Depto. Cochinoca, Abra Pampa, Cerro Huancar. 23-I1948. Cabrera 9425 (SI); Sierra de Quichagua, 3940 m. 26III-2006. Peterson et al. 19559 (SI); Casa linda, peñas inmediaciones (al SE) del pueblo, ojo de agua. 23-XII-1980. Ana C. 2298 (SI); Abra Pampa, Estación Experimental del INTA, 3470 m. 15-III-1978. Arenas et al. s. n. (SI); Abra Pampa, Cerro Huancar, 3500 m. 17-II-1963. Cabrera 15273 (SI); Abra Pampa, Cerro Huancar. 20-I-1976. Cabrera et al. 27401 (SI); Abra de la Pampa. 20-I-1976. Cabrera et al. 29401 (SI); Depto. El Carmen, Abra de Lipán, 4100 m. 26III-1992. Cabrera et al. 34841 (SI); Depto. Humahuaca, Mina Aguilar. 4600 m. 15-I-1948. Cabrera 9261 (SI); Mina Aguilar, 4500 m, 11-I-1968. Cabrera et al. 18975 (SI); Cerro Aguilar. 4400 m. 25-II-1953. Hunzinker et al. 6214 (SI); Camino a Palca de Aparzo, a $28 \mathrm{~km}$ de Humahuaca, $4200 \mathrm{~m}$. 26-II-1983. Hunzinker et al. 10422 (SI), Sierra del Aguila, 4000 m. 03-IX-1929. Venturi 8734 (SI); entre Palca de Aparzo y Sta. Ana, 3870 m. 11-II-2007. Zuloaga et al. 9180* (SI); Depto. Rinconada, Minas Pirquitas. 23-II-1980. Arenas 1853 (SI); Laguna de Pozuelos. 3690 m. 12-II-1995.

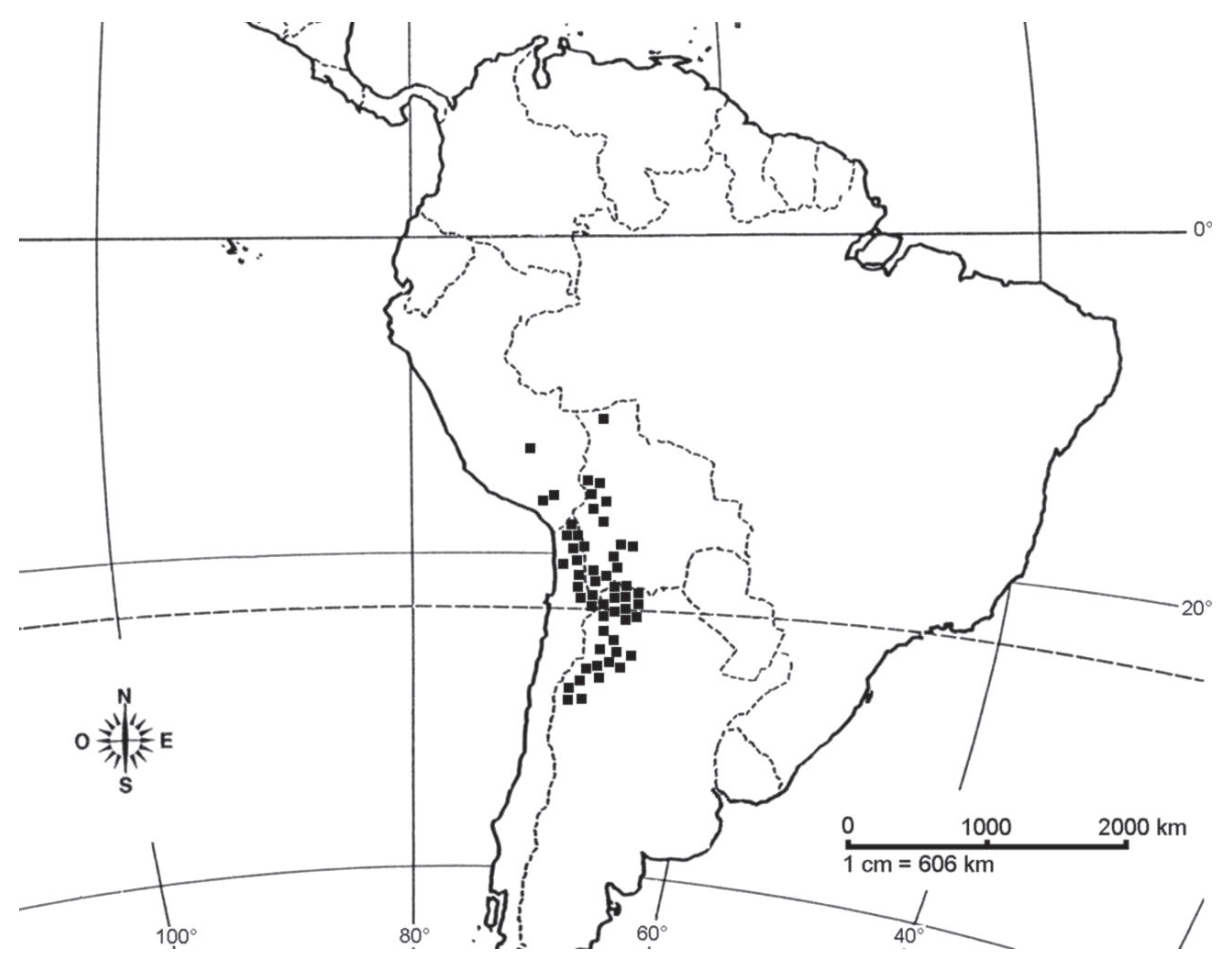

Figura 4. Distribución geográfica de Festuca chrysophylla Phil.

Figure 4. Geographical distribution of Festuca chrysophylla Phil. 
Deginani et al. 611 (SI); $67 \mathrm{~km}$ al S de Mina Pirquitas, Laguna de Vilama, 4530 m. 14-II-1999. Morrone et al. 2555 (SI); Pan de Azúcar. 03-III-1966. de la Sota 4220 (SI); Depto. Santa Catalina, Firtz Claren 11615 (SI); Depto. Susques, Campo Amarillo. 16-II-1980. Cabrera et al. 31789 (SI); N of Susques, ENE Paso de Jama, SE of Cerro Tocol. 4132 m. 26-III-2006. Peterson 19547 (SI); Paso de Jama, límite con Chile, 4250 m. 23-II-2009. Zuloaga et al. 11143* (SI); Depto. Tumbaya, Abra de Lipán. 4000 m. 24-III-1979. Cabrera et al. 30528* (SI); subida al Abra de Lipán. 3800 m. 15-II-1980. Cabrera et al. 31676(SI); camino de Purmamarca al Abra de Lipán, Abra Blanca, 3900 m. 17-II-1985. Kiesling 5307 (SI); Alto del Morado, entre Purmamarca y Tres Morros, 4175 m. 18-II-2008. Zuloaga et al. 10200 (SI); Abra de Lipán, 4200 m. 17-I-1988. Zuloaga et al. 3558 (SI); Depto. Valle Grande, Caspalá, cumbres a 4400 m, comienzo del declive occidental.03-III-1940. Burkart et al. 11825* (SI); Depto. Yavi, Abra de Lizoite, 4450 m. 01-III-1970. Fabris 7778* (SI); Depto. sin consignar, Cerca del Abra Yampaite, camino a Casa Linda. 18-I-1948. A. L. Cabrera 9308 (SI). Prov. La Rioja, Depto. General Belgrano, Sierra de Famatina, Mina La Mejicana, 4000 m. 03-II-1995. Ruthsatz 9138* (SI); Mina La Mejicana, Cueva de Pérez, 3850 m. 20-IV-1995. Biurrun 4139 (SI); entre Los Corrales y Cueva de Pérez, 3800 m. 13-I-1976. Cabrera et al. 27178* (SI); entre Los Corrales y Cueva de Pérez, 3500 m. 13-I1976. Cabrera et al. 27204 (SI); Cueva de Pérez, Sierra de Famatina, 4200 m. 15-I-1947. Hunzinker et al. 1943 (SI). Prov. Salta, Depto. Los Andes, San Antonio, Alto de Chorrillos, 14560 m. 27-I-1949. Cabrera 122 (SI); Hwy 51 W of San Antonio de los Cobres, just S of pass to Jujuy, 4301 m. 25-III-2006. Peterson et al. 19545 (SI); ruta 51, de San Antonio de los Cobres a Viaducto La Polvorilla, 4170 m. 18-II-2002. Cialdella et al. 419 (SI); ruta 51, $8 \mathrm{~km}$ después del desvío a Santa Rosa de Los Pastos Grandes, 4410 m. 18-II-2002. Cialdella et al. 431 (SI); ruta 51, Alto Chorrillo, 4530 m. 24-II-2009. Zuloaga et al. 11179* (SI); desvío de la ruta 51 hacia viaducto La Polvorilla, $3950 \mathrm{~m}$. 13-II-2007. Zuloaga et al. 9310* (SI); Depto. Rosario de La Frontera, Laguna Seca, cerro del Cajón, 4200 m. 15-II1914. Rodriguez 1351(a) (SI); Depto. Santa Victoria, ruta Prov. 145 de El Cóndor a Nazareno, Abra del Cóndor, 4500 m. 16-II-2009. Zuloaga et al. 10802 (SI); Depto. sin consignar, Quebrada de Paizaite, 3500 m. 02-II-1943. Meyer 4899 (SI). Prov. Tucumán, Depto. Tafí del Valle, cumbres Calchaquíes, 4300 m. 14-III-1984. Gómez-Sosa et al. 182* (SI); El Pelao. 15-III-1912. Rodríguez 425* (SI); cumbre Calchaquí, 4400 m. 30-I-1933. Parodi 10832 (SI). BOLIVIA, Depto. La Paz, Prov.Aroma, South of La Paz on Hwy 1 towards Oruro, 6,7 $\mathrm{km}$ of Villa Loza on road to Urmiri and Spahaqui, $4040 \mathrm{~m}$. 04-III-1993. Peterson et al. 12626* (SI); Prov. Camacho, Puerto Acosta, 4 km hacia La Paz, 3860 m. 06-IV-1982. Beck 7663* (SI); Prov. Los Andes, $1 \mathrm{~km}$ NW from Tambillo, 3750 m. 31-III-1981.
Renvoize et al. 4128* (SI); Prov. Ingavi, alrededores de Viacha, 3900 m. 01-II-1979. Ceballos et al. 110 (SI). Depto. Oruro, Prov. Cercado, sistema de dunas a la salida de la ciudad, 3800 m. 01-II-1979. Ceballos et al. 205 (SI); Prov. Saucarí, comunidad Kollpuma, 60 km al SW de Oruro, 3712 m. 12-II-1992. Ayala 4* (SI). Depto. Potosí, Prov. Linares, al norte de Cucho Ingenio, $4185 \mathrm{~m}$. 06-IV-1997. Cocucci et al. 845 (SI); Prov. Quijarro, $14 \mathrm{~m} \mathrm{~S}$ of Severuyo on road towards Uyuni. 3630 m. 09-III-1993. Peterson et al. 12797* (SI); Prov. Sud Lípez, Cerro Tapaquilcha, 4600 m. 11-IV1980. Limbermann 168* (SI); región de Laguna Colorada, lomas de Toloncejara, 4870 m. 20-IV-1991. Navarro 420* (SI); región de Laguna Colorada, laderas orientales del Cerro Negro, 4450 m. 24-IV-1991. Navarro 476* (SI); región de Laguna Colorada, Cerro Pabellón, 4860 m. 18-IV1990. Navarro 479 (SI); N of Río Mulatos on road towards Challapata, 3650 m. 10-III-1993. Peterson et al. 12808* (SI); Prov. Tomás Frías, Khare-Khare, arriba de la fundición de Karachipampa, 4400 m. 13-III-1988. Schulte 175 (SI). Depto. Tarijá, Prov. José María Avilés, Arenales, cerca de Copacabana, 3750 m. 06-III-1998. Beck 23694* (SI). CHILE, Región de Antofagasta, Prov. Antofagasta, $4680 \mathrm{~m}$. 22-III-2001. Peterson et al. 15602 (SI); km 31,5 East from San Pedro de Atacama on paved HWY towards Argentine border at Paso de Jama, $3985 \mathrm{~m}$. 16-III-2001. Peterson et al. 15508* (SI); km 33,5 East from San Pedro de Atacama on paved HWY towards Argentine border at Paso de Jama, 4120 m. 16-III-2001. Peterson et al. 15510* (SI); km 43 on road from San Pedro to Paso Jama road, then south 1,5 km on Cerro Toto or Chascon de Purico, 4740 m. 16-III-2001. Peterson et al. 15521* (SI); Cuesta de Chita on road from Capana to el Tatío, km 34, 3995 m. 19-III-2001. Peterson et al. 15568* (SI); sin localidad consignada, 4400 m. 17-III2001. Peterson et al. 15535 (SI); $6 \mathrm{~km}$ south of El Tatío "Hotel" at km 80 on road north from San Pedro de Atacama to El Tatío, 4396 m. 19-III-2001. Peterson et al. 15570* (SI); Prov. El Loa, San Pedro de Atacama, Mountaraqui, vega Zorras, 4250 m. 14-III-1992. Ruthsatz 8243* (SI). Región de Arica y Parinacota, Prov. Arica, $22 \mathrm{~km}$ E of Zapahuira at Portezuelo Chapiquiña, 4460 m. 02-IV-2001. Peterson et al. 15731 (SI); $22 \mathrm{~km} \mathrm{E}$ of Zapahuira at Portezuelo Chapiquiña, 4460 m. 02-IV-2001. Peterson et al. 15732 (SI); Las Cuevas, 4400 m. 05-V-1977. Escobar 118 (SI); termas Chiriguaya, 4480 m. 05-IV-2001. Peterson et al. 15767* (SI); Prov. Parinacota, Volcán Tacora, Chislluma, 4500 m. 01-IV-1926. Werdermann 1161* (SI); 133 km E of Arica at Termas Jurasi above Putre, 4040-4150 m. 03-IV2001. Peterson et al. 15748 (SI); $133 \mathrm{~km} \mathrm{E} \mathrm{of} \mathrm{Arica} \mathrm{at}$ Termas Jurasi above Putre, 4040-4150 m, 03-IV-2001. Peterson et al. 15745* (SI), $3 \mathrm{~km} \mathrm{~N}$ of Parinacota, 04-IV2001. Peterson et al. 15755 (SI); sur de Laguna Chungará, 4750 m. 31-III-1992. Ruthsatz 8437 (SI); 15 km E of Zapahuira on road towards Guallatiri, 4156 m. 01-IV-2010. Peterson et al. 15713 (SI); $26 \mathrm{~km} \mathrm{E} \mathrm{of} \mathrm{Zapahuira} \mathrm{on} \mathrm{top} \mathrm{of}$ 
Cerro Champiquiña, 4810 m. 01-IV-2001. Peterson et al. 15723 (SI). Región de Tarapacá, Prov. Tamarugal, 82 km NE of Huara on HWY A-55 towards Colchane, 3890 m. 25III-2001. Peterson et al. 15630 (SI); $92 \mathrm{~km}$ NE of Huara on road towards Colchane, 4600-4830 m. 26-III-2001. Peterson et al. 15633 (SI); $110 \mathrm{~km} \mathrm{NE}$ os Huara on road towards Colchane, 4120 m. 27-III-2001. Peterson et al. 15662* (SI); $110 \mathrm{~km}$ of Huara on road towards Colchane, $4210 \mathrm{~m}$. 27-III2001. Peterson et al. 15660 (SI); $66 \mathrm{~km} \mathrm{NW}$ of Colchane on road towards Chilcayo at Porto Capitán, just E of Cerro Capitán, 4464 m. 28-III-2001. Peterson et al. 15674* (SI); Cord. Co. Columtusca, Apacheta, 4600 m. 01-III-1926. Werdermann $1072 *$ (SI); $94 \mathrm{~km} \mathrm{NE}$ of Huara on road towards Colchane, 4078 m. 26-III-2001. Peterson et al. 15637 (SI). PERÚ Cusco Espinar Chisiccata, Yauri. 4000 m. 28-III-1987. Núñez 7627* (SI).

Festuca wedermannii St.-Yves, Candollea 3: 301. 1927. TIPO: Chile. Provincia de Coquimbo, Baños del Toro, ca. 3600 m, dic. 1923, E. Werdermann 209 (lectotipo G00099477, foto!, designado por Alexeev, Bot. Zhurn. (Moscow \& Lenigrad): 69: 349 (1984); duplicados B-10-0250132, foto!, BM000884685, E00254713, foto!, F-549333, foto!, K000433664, foto!, LIL-44254!, S05-5095, foto!, MO910593, foto!, SI-001838!, US-1498126, foto!). Fig. 5.

Hierba perenne, densamente cespitosa, hojas principalmente basales, que mayormente no superan el medio de la altura de planta. Cañas floríferas de 20-35(40) cm de alto. Vainas dísticas, flojas, abiertas en su tercio superior, pubérulas, a veces con pelos en el margen y zona ligular, papiráceas a membranáceas, hialinas, blanquecinas a pajizas, cuello con pulvínulo emergente, oscurecido, cortamente piloso a glabro. Lígulas de 2-3,5 $\mathrm{mm}$ de largo en la parte media, decurrente con la vaina, bilobulada. Láminas $6-10 \mathrm{~cm}$ de largo, juncoides, rígidas, terminadas en un mucrón, arqueadas, superficie abaxial escabrosa, superficie adaxial y el margen densamente pilosos. Panículas de 4-12 cm de largo, lineares; ramas adpresas al raquis, escabrosas. Espiguillas 6-10 mm de largo, 3-5 floras. Glumas desiguales, lanceoladas, escariosas, margen frecuentemente eroso, la superior alcanza el medio del antecio contiguo, cortamente ciliadas en el margen y escabrosas en el ápice y dorso; la inferior 2,8-3,5 mm de largo, de ápice agudo, 1 nervia; la superior 3,7-4,1 mm de largo, aovada-lanceolada, de ápice agudo a comúnmente obtuso, erosa en el ápice, 3 nervia. Lema 4,5-6 $\mathrm{mm}$ de largo, glabra, lanceolada, de ápice agudo, escariosa. Pálea 4-5,2 mm de largo, membranácea, hialina a blanquecina, carenas glabras. Anteras de 2,7-3,5 $\mathrm{mm}$ de largo. Lodículas 0,5-0,6 $\mathrm{mm}$ de largo, lobuladas, glabras. Ovario híspido en el ápice. Cariopse ovoide.

Caracteres histofoliares en corte transversal (Fig. 3C y 3D). Láminas permanentemente plegadas, conduplicadas, de transcorte elíptico, en ocasiones ligeramente triangular (Cabrera 3521) con semi-láminas simétricas, de 0,7-1,5 $(-1,8) \mathrm{mm}$ de diámetro, superficie adaxial con costillas marcadas de redondeadas a agudas asociadas a todos los haces vasculares, surcos notorios entre haces vasculares; superficie abaxial lisa o algo irregular pero sin distinción en surcos y costillas; haces vasculares libres distribuidos aproximadamente equidistantes de ambas epidermis, 3 haces primarios alternando con dos haces menores, todos los haces vasculares rodeados por una vaina esclerenquimática continua de células engrosadas en paredes radiales y tangenciales internas, en algunos casos con una segunda vaina externa irregular de células parenquimáticas pequeñas de paredes delgadas que se diferencian por su tamaño del resto del mesófilo (Kiesling 8664, Teillier 4526, Wedermann 209); clorénquima compacto de disposición no radiada, con células globosas, isodiamétricas, de tamaño regular; esclerénquima subepidérmico escaso, generalmente discontinuo, formando delgados casquetes abaxiales asociados a los haces vasculares, extendiéndose escasamente hacia los márgenes, o formando un delgado anillo abaxial continuo de 1-3 capas (Ruthsatz 9164), esclerénquima ausente en la cara adaxial; epidermis abaxial en corte transversal de células muy engrosadas de mayor diámetro que la epidermis adaxial, con asperezas especialmente en las zonas opuestas a los haces vasculares, epidermis adaxial con células de pared tangencial externa algo convexa, engrosada, con estomas ubicados en las zonas de los surcos, macropelos unicelulares en zonas costales e intercostales, células buliformes escasamente diferenciadas, en número de 3-5, ubicadas en la profundidad de los surcos.

EPIDERMIS FOLIAR EN VISTA TOPOGRÁFICA. Epidermis abaxial con escasa diferenciación entre zonas costales e intercostales, células largas de contorno rectangular, elongadas, 2-6 veces más largas que anchas, con paredes anticlinales longitudinales paralelas, muy engrosadas, con ondulaciones muy profundas, fuertemente corrugadas y paredes anticlinales transversales engrosadas y rectas; aparatos estomáticos ausentes; asperezas (ganchos y/o aguijones) presentes, de moderados a abundantes, principalmente en zonas costales, de base oval, ocasionalmente con paredes silicificadas; micropelos ausentes; macropelos ausentes o en ocasiones presentes (Gajardo 7), en este caso algo más cortos que los de la epidermis adaxial (300-400 $\mu \mathrm{m}$ largo), unicelulares, de base simple, cuerpos silíceos costales e intercostales solitarios alternando con células largas, verticalmente elongados, rectangulares o más estrechos en la zona media. Epidermis adaxial con zonas costales e intercostales bien distinguibles, células largas semejantes a las de la epidermis abaxial, en ocasiones con paredes transversales menos sinuosas (Teillier 4526); aparatos estomáticos siempre presentes en zonas intercostales, con células subsidiarias de paredes redondeadas, en forma de 

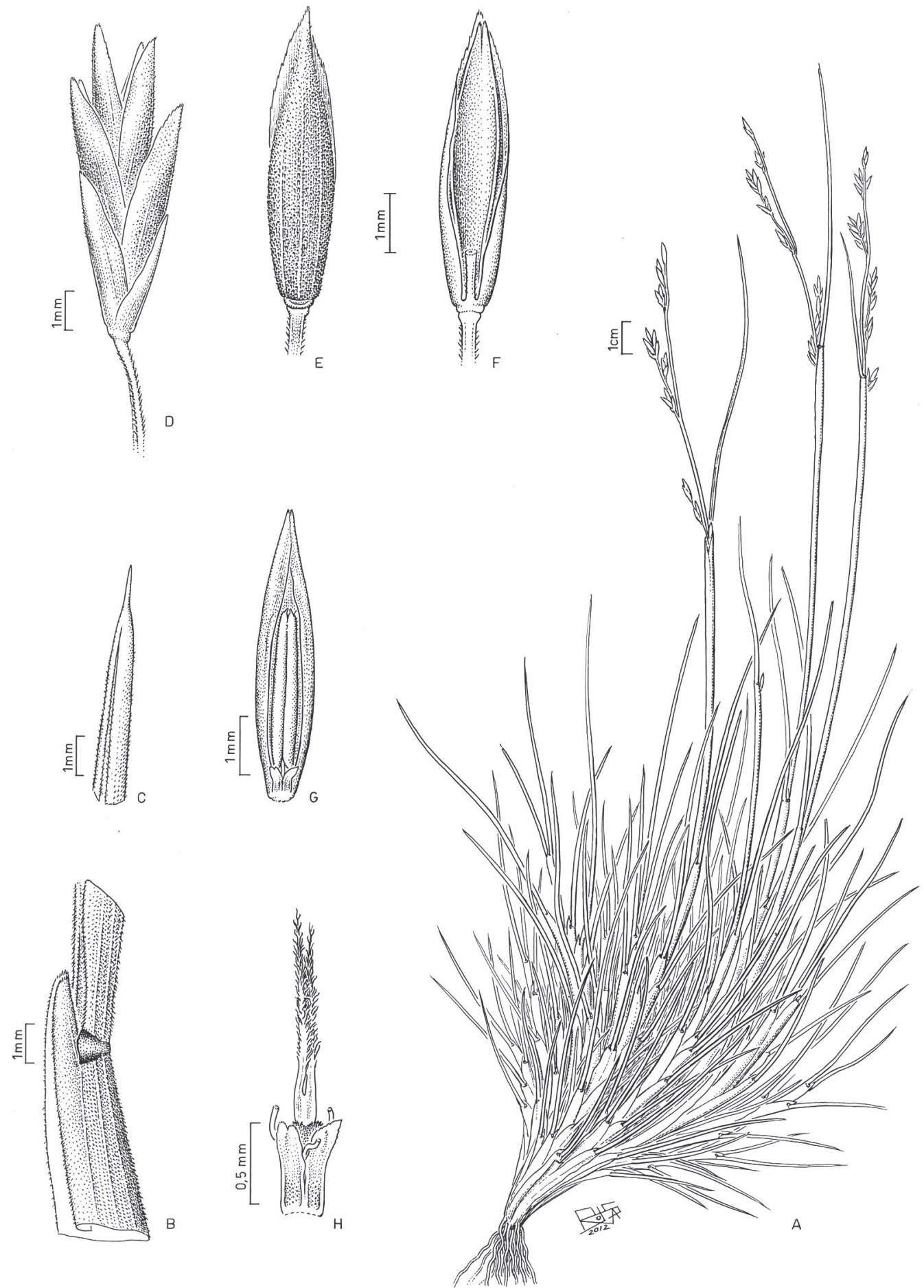

Figura 5. Festuca werdermannii St.-Yves, Werdermann 209 (isolectotipo). A. Hábito; B. Zona ligular; C. Ápice de lámina atenuado en mucrón; D. Espiguilla; E. Antecio vista dorsal; F. Antecio vista ventral; G. Pálea con lodículas y anteras; H. Lodículas y ovario.

FIgure 5. Festuca werdermannii St.-Yves, Werdermann 209 (isolectotype). A. Habit; B. Ligular zone; C. Apex of the blade with mucron; D. Spikelet; E. Anthecium dorsal view; F. Anthecium ventral view; G. Palea with lodicules and anthers; H. Lodicules and ovary. 
domo bajo; asperezas presentes, en particular con mayor densidad en las zonas costales, semejantes a las de la epidermis abaxial; cuerpos silíceos costales e intercostales semejantes a los de la epidermis abaxial, o longitudinalmente elongados acompañando a los lados de los estomas (Kiesling 8664); macropelos siempre presentes, unicelulares, de base simple, rectos a ligeramente curvados, de hasta $700 \mu \mathrm{m}$ de largo.

DistribuCión y hábitat (Fig. 6): En Chile se distribuye en el norte de la Región de Coquimbo, entre 3.200 y 3.700 m, crece en zonas xerofíticas y mesofíticas cerca de arroyos o en vertientes donde la humedad es abundante; es una especie de distribución reducida en la alta cordillera andina (Matthei 1982). En Argentina se distribuye en el noroeste, en San Juan y La Rioja, a orillas de arroyos, vegas y regiones andinas, entre 3.200 y $3.980 \mathrm{~m}$ de altitud.

Observaciones: Los caracteres distintivos de la especie son las láminas mayormente basales, la mayoría hasta la mitad de la planta, plantas de 20-35(-50) cm de alto. Vainas hialinas, flojas, pajizas a blanquecinas; lígula larga, de
$(3,5) 2-3 \mathrm{~mm}$ de largo en la parte media, decurrente con la vaina, bilobulada, no auriculada, cuello glabro, pulvinado, sobreelevado, oscurecido. Glumas lanceoladas, la inferior aguda, la superior ancha, obtusa a aguda, erosa en el ápice, lema escariosa, lanceolada, aguda.

Especímenes examinados: ARGENTINA, Prov. La Rioja, Depto. Chilecito, Sa. de Famatina, Río Amarillo, 3400 m. 4-II-1995. Ruthsatz 9164* (SI); Prov. San Juan, Depto. Iglesia, río Las Taguas, parcela 1, arroyo Las Yaretas. 06-I-2000. Gajardo 7* (SI); Depto Calingasta, N de Gendarmería, río Blanco, 3750 m. 22-01-1995. Kiesling et al. 8664 (SI); río Turbio. 23-01-1995. Kiesling et al. 8677 (SI); confluencia de río Turbio y río Las Taguas. 20-3-1998. Kiesling 9056 (SI); río Las Taguas, quebrada del río Turbio, cerca vega del ex campamento de Mina Aguilar, 3800 m. 21-01-1999. Kiesling et al. 9223 (SI); zona del río La Taguas, quebrada Potrerillos, 3700 m. 04-02-2000. Teillier 4526* (SI). CHILE, Región de Coquimbo, Prov. Coquimbo, cordillera de Coquimbo, Baños del Toro, río Toro, $3300 \mathrm{~m}$. 18-01-1936. Cabrera 3521*. (SI); Baños del Toro, $3600 \mathrm{~m}$. 12-1923. Werdermann 209* (SI, isolectotipo).

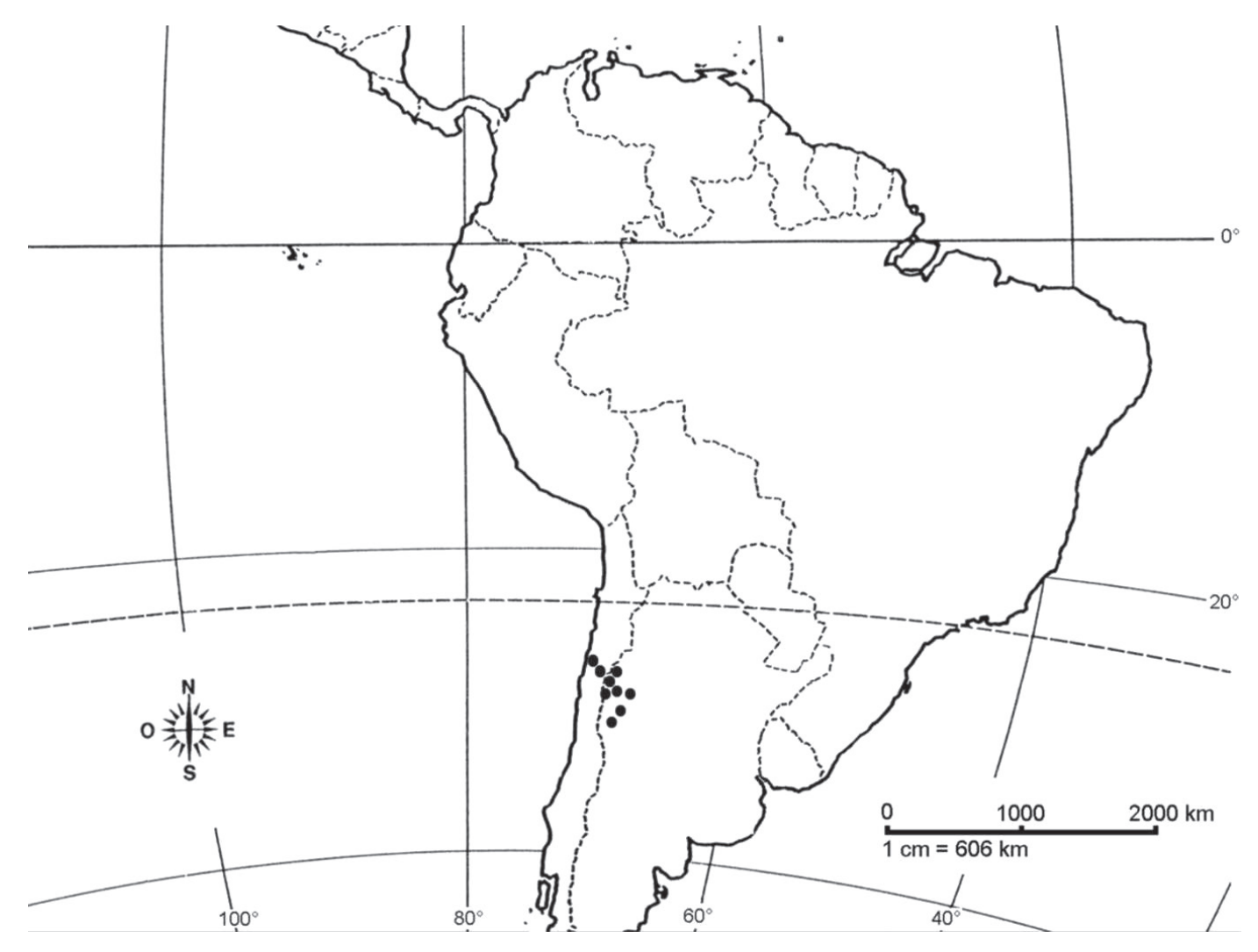

Figura 6. Distribución geográfica de Festuca wedermannii St.-Yves.

FIgURE 6. Geographical distribution of Festuca werdermannii St.-Yves. 


\section{AGRADECIMIENTOS}

A los curadores de los herbarios, Mélica Muñoz y Gloria Rojas (SGO), a Alicia Marticorena y Rosa Montero (CONC). A Víctor Finot por la ayuda brindada. A los revisores por sus valiosas sugerencias. A Vladimiro Dudas y Francisco Rojas por las ilustraciones. Los autores son investigadores de CONICET, Argentina.

\section{BIBLIOGRAFÍA}

AleXeev, E.B. 1984. The new taxa of the genus Festuca (Poaceae) from Colombia and Ecuador. Botanicheskii Zhurnal 69: 1543-1551.

Alexeev, E.B. 1986. Festuca L. (Poaceae) in Venezuela, Colombia \& Ecuador. Novosti Sistematiki Vysshikh Rastenii 23: 5-23.

Balzarini, M.G., L. González, M.Tablada, F. Casanoves, J.A. Di Rienzo \& C.W. Robledo. 2008. Infostat. Manual del Usuario. Editorial Brujas, Córdoba. 336 pp.

Briceño, B. \& G. Morillo. 1994. El género Festuca L. (Poaceae) en los páramos de Mérida. Ernstia 4: 73-88.

Catalán, P. \& J. Müller. 2012. Festuca. En: A. M. Anton \& F. O. Zuloaga (eds.), Flora de Argentina, Vol. 3, Tomo II. 219 250 pp. Gráficamente Ediciones, Córdoba, Argentina.

Cabrera, A.L. 1957. La vegetación de la Puna Argentina. Revista de Investigaciones Agrícolas 11(4):317-412.

Di Rienzo, J.A., F. Casanoves, M.G. Balzarini, L. González, M. TABLADA \& C.W. RoBledo. 2011. InfoStat, versión 2011, Grupo InfoStat, FCA, Universidad Nacional de Córdoba, Argentina. URL http://www.infostat.com.ar

Denham, S. \& S. Aliscioni. 2010. Species delimitation in the Sporobolus aeneus complex (Zoysieae, Chloridoideae, Poaceae) using the phylogenetic species concept. Taxon 59(6): 1765-1782.

Dubcovsky, J. \& A.J. Martínez. 1991. Chromosome complement and nucleoli in the Festuca pallescens alliance from South America. Canadian Journal of Botany 69(12): 2756-2761.

ELLIS, R.P. 1976. A procedure for standardizing comparative leaf anatomy in the Poaceae. I. The leaf-blade as view in transverse section. Bothalia 12(1): 65-109.

ElLIS, R.P. 1979. A procedure for standardizing comparative leaf anatomy in the Poaceae. II. The epidermis as seen in surface view. Bothalia 12(4): 641-671.

Escobar, I., E. Ruiz, V. Finot, M.A. Negritto \& C.M. Baeza. 2011. Revisión taxonómica del género Eragrostis Wolf en Chile, basada en análisis estadísticos multivariados. Gayana Botánica 68(1): 49-85.

Finot, V.L., C. Marticorena, J.A Barrera, M. Muñoz-Schick \& M.A. Negritto. 2009. Diversidad de la familia Poaceae (Gramineae) en la Región del Bío-Bío, Chile, basada en colecciones de herbario. Gayana Botánica 66(2): 134-157.

Gower, J.C. 1971. General coefficient of similarity and some of its properties. Biometrics 27: 857-874.

Henderson, A.J. 2005. A multivariate study of Calyptrogyne (Palmae). Systematic Botany 30 (1): 60-83.

Hiтchсоск, A.S. 1927. The grasses of Ecuador, Peru, and Bolivia.
Contributions from the United States National Herbarium 24(8): 291-556.

INFANTES, J. 1952. Estudio botánico, químico y fitogeográfico de 27 especies del género Festuca. Revista de Ciencias (Lima) 54: 76-154.

Laegaard, S. 1999. Festuca. En: P.M. Jørgensen \& S. León-Yáñez (eds.), Catalogue of the Vascular Plants of Ecuador, Vol. 75, pp 819-820. Missouri Botanical Garden Press, Saint Louis.

MacBride, J.F. 1936. Gramineae. En: B.E. Dahlgren (ed.), Flora of Peru. Field Museum of Natural History, Botanical Series XIII Part 1. pp 96-261. Chicago.

Marticorena, C. \& M. Quezada. 1985. Catálogo de la flora vascular de Chile. Gayana Botánica 42: 5-157.

Matthei, J.O. 1982. El género Festuca (Poaceae) en Chile. Gayana Botánica 37: 1-64.

Metcalfe, C.R. 1960. Anatomy of the Monocotyledons. I. Gramineae, Clarendon Press, Oxford. 731 pp.

NicorA, E.G. 1978. Gramineae. En: M.N. Correa (ed.), Flora Patagónica, parte III. Colección Científica del I.N.T.A. Buenos Aires. 1-581 pp.

NicorA, E.G. \& Z.E. Rúgolo de Agrasar. 1987. Los géneros de gramíneas de America Austral, $2^{\text {a }}$ edición, Hemisferio Sur, Buenos Aires. 611 pp.

Parodi, L.R. 1935. Notas sobre gramíneas argentinas. Physis 11: 497-500.

Parodi, L.R. 1953. Las especies de Festuca de la Patagonia. Revista Argentina de Agronomía. 20: 177-229.

PILGER, R. 1906. Gramineae andinae. III. Botanische Jahrbücher für Systematik, Pflanzengeschichte und Pflanzengeographie 37: 504-517.

Rangel Churio, O.J. 2000. Colombia Diversidad Biótica III. La región de vida paramuna. Editorial Unibiblos, Universidad Nacional de Colombia, Bogotá. 902 pp.

Rangel Churio, O.J., P.D.C. Lowy \& M.P. Aguilar. 1997. Colombia Diversidad Biótica II. I.C.N., Universidad Nacional de Colombia, Bogotá. 436 pp.

Renvoize, S.A. 1998. Grasses of Bolivia. Kew, London. 664 pp.

Ruzin, S.E. 1999. Plant microtechnique and microscopy. University Press, Oxford. 322 pp.

SAINT-Yves, A. 1927. Contribution a l'étude des Festuca (subg. Eu-Festuca) de l'Amérique du Sud. Candollea. 3: 151-315.

Soreng, R.J., G. Davidse, P.M. Peterson, F.O. Zuloaga, E.J. Judziewicz, T.S. Filgueiras \& O. Morrone. 2003. Catalogue of New World Grasses (Poaceae). Contributions from the United States National Herbarium 48: 1-730.

Stančík, D. 2001. New records of the genus Festuca L. (Poaceae) for Colombia and Venezuela. Caldasia 23: 337-339.

Stanč́́, D. 2003a. The genus Festuca (Poaceae) in Colombia. Darwiniana 41(1-4): 93-153.

Stančík, D. 2003b. New endemic taxa in Festuca (Poaceae) from Colombian Sierra Nevada de Santa Marta. Preslia 75: 1-9.

Stančík, D. \& P.M. Peterson. 2007. A revision of Festuca (Poaceae: Loliinae) in South American Paramos. Contributions from the United States National Herbarium 56: 1-184.

StuCKert, T. 1906. Segunda contribución al conocimiento de las gramináceas argentinas. Anales del Museo Nacional de Historia Natural de Buenos Aires 3(6): 409-555.

ThIERS, B. [continuously updated, accessed 2012] Index 
Herbariorum: a global directory of public herbaria and associated staff. New York Botanical Garden's Virtual Herbarium, URL: http://sweetgum.nybg.org/ih.

Tovar, O. 1972. Revisión de las especies peruanas del género Festuca, Gramineae. Memorias del Museo de Historia Natural "Javier Prado" 16: 1-93.

TürPe, A.M. 1969. Las especies argentinas de Festuca. Darwiniana. 15: $189-283$.
TÜRPE, A.M. 1998. Novedades en gramineae para la flora argentina. Hickenia 57(2): 267-272.

Zuloaga, F.O., O. Morrone \& M. Belgrano (eds.). 2008. Catálogo de las Plantas Vasculares del Cono Sur. I. Pteridophyta, Gymnospermae y Monocotyledoneae. Missouri Botanical Garden Press, Saint Louis. pp. 1-983.

APÉNDICE I. Listado de caracteres usados en el análisis estadístico y su codificación.

APPENDIX I. List and coding of characters used in the statistic analysis.

ANATÓmicos

Discretos. Número de haces vasculares primarios. Número de haces vasculares secundarios. Número de haces vasculares terciarios. Número de capas de células del anillo esclerenquimático. Doble-estado. Zona costal adaxial asociada a: haz primario (0), haces menores (1). Zona costal del haz central: redondeada (0), angular (1). Epidermis abaxial y esclerénquima: no diferenciadas (0), diferenciadas (1). Haz central adaxialmente: libre (0), trabado (1). Haz menor contiguo al central: libre (0), trabado (1). Haz primario contiguo al central: traba abaxial (0), traba adaxial (1). Haces menores asociados a: zona costal (0), zona intercostal (1). Pares sílico-suberosos: ausentes (0), presentes (1). Asperezas o aguijones en superficie abaxial: ausentes (0), presentes (1).

Morfológicos

Continuos. Longitud de la panoja (cm). Longitud del antecio basal (mm). Longitud gluma inferior ( $\mathrm{mm})$. Longitud gluma superior $(\mathrm{mm})$. Longitud lígula $(\mathrm{mm})$. Alto planta $(\mathrm{cm})$. Longitud lámina $(\mathrm{cm})$. Discretos. Número de nudos en la zona de elongación: 1 (0), 2 (1). Ápice del ovario: glabro (0), con pelos (1). Ángulo de disposición de las láminas con respecto a la caña: menor de $45^{\circ}(0)$, mayor de $46^{\circ}(1)$.

Recibido: 09.11.12

Aceptado: 22.01.13 